\title{
F045
}

\section{Simultaneous Vertical Seismic Profiling in Two Wells and the Virtual Source Method; Perth Basin Western Australia}

\author{
M. AlMalki* (Curtin University), B. Harris (Curtin University) \& C. Dupuis \\ (Curtin University)
}

\section{SUMMARY}

Vertical seismic profiling experiments have been completed simultaneously in two approximately $400 \mathrm{~m}$ deep wells. The field experiment provided the data necessary to examine and compare the virtual source method VSM and zero-offset vertical seismic profiling ZVSP. Both methods provide an improved understanding of the acoustic heterogeneity of the weakly consolidated sandstone aquifers in the Perth Basin, Western Australia. Acquisition parameters for the simultaneous VSP experiment were designed to optimize the chance of removing unnecessary wave modes. The number of hydrophones deployed is of considerable important if a detailed velocity distribution between the two vertical wells is to be achieved. Low ray coverage cells tend to have incorrect velocity values. We examine potentially serious statics problems that can result from using a $1400 \mathrm{~kg}$ weight dropped source in dry unconsolidated sand. We provide an example of how these problems can be successfully managed by measuring simultaneously in two wells. We comparing the velocities distributions obtained from VSM and ZVSP methods with sonic and gamma wire-line logs. Both methods are demonstrated to be effective for obtaining detailed velocity distributions. In particular the virtual source method is highly promising for imaging velocity distribution between two vertical wells where ray coverage is high. 


\section{Vienna |'11}

\section{Introduction}

We have completed a field experiment designed to trial the virtual source methods VSM using simultaneous acquisition of seismic data in hydrophones set in two vertical wells. The wells were $25 \mathrm{~m}$ apart and approximately $400 \mathrm{~m}$ deep. The source was a $1400 \mathrm{~kg}$ weight dropped from a height of 1.5 $\mathrm{m}$. The experiment was completed at the Mirrabooka Trial Aquifer Storage and Recovery (ASR) site, Perth, Western Australia. Twenty four hydrophones with $10 \mathrm{~m}$ spacing were deployed in each monitoring well (i.e. M345-109 and M345-408). All 48 hydrophones were active during acquisition. One objective of the dual well vertical seismic profiling experiment was to assess the field parameters and processing required for application of the VSM method. A further objective was to create and compare accurate velocity models using the Virtual Source and the simultaneous ZVSP methods. The outcome will help improved understanding heterogeneity within key aquifers in the Perth Basin Western Australia.

\section{Zero-offset vertical seismic profile experiment:}

The source for the detailed zero offset VSP was located $32 \mathrm{~m}$ away from the, $100 \mathrm{~mm}$ diameter fibreglass reinforced plastic (FRP), water monitoring well M345-408. Twenty four hydrophones deployed were in M345-408 over a fixed depth range from $120 \mathrm{~m}$ to $350 \mathrm{~m}$ below surface. Hydrophone spacing was $10 \mathrm{~m}$. The borehole string in M345-408 was stationary during the detailed acquisition in M345-109.

The 100mm diameter FRP vertical water monitoring well M345-109 was located $12.5 \mathrm{~m}$ away from the source location and initially hydrophones were deployed over the depth interval 170 to $400 \mathrm{~m}$ below ground level. To acquire the detailed ZVSP data, the hydrophone string in M345-109 was moved up 10 times by $1 \mathrm{~m}$ each time to provide 240 hydrophone positions between 160 and $400 \mathrm{~m}$. High coverage was required for processing. In particular high coverage is necessary to improve tube waves removal and static corrections.

Soil compaction at the impact source plate can be as much as $40 \mathrm{~cm}$. This is a significant problem as velocities at the shot point are very slow (less than $400 \mathrm{~m} / \mathrm{sec}$ ) and large errors in travel time will negatively affect processing. However as the hydrophones in 408 are not moved the travel times to this well should remain constant. Any variation in travel time at M345-408 can be used for a highly accurate travel time correction (static correction) for records in M345-109. That is, M345-408 can be uses as a reference to calibrate the arrival time for each shot at M345-109.

Static corrections are essential if wave-field separation is to be successful. This is particularly the case for processing of hydrophone ZVSP where removal of tube waves is often problematic. After static correction tube waves become aligned and can be suppressed by processing in F-K domain. Figure 1A and 1B shows all 240 traces recorded in M345-109 as common receiver gathers. Note the considerable improvement in definition of down going and up-going tube and $\mathrm{P}$ waves after applying static correction in the f-k domain. After static correction the down-going $\mathrm{P}$ wave can be seen at frequencies up to $300 \mathrm{~Hz}$ which is important if high resolution imaging is to be achieved.

\section{Virtual source experiment:}

The virtual cross-well data was acquiring simultaneously in the same hydrophones in vertical boreholes M345-109 and M345-408. Borehole M345-109 contained 24 active hydrophones deployed between $170 \mathrm{~m}$ to $400 \mathrm{~m}$. Borehole M345-408 contained 9 active hydrophones distributed in the depth range $180 \mathrm{~m}$ to $340 \mathrm{~m}$. A surface line of shots was generated at 149 source positions spaced at $2 \mathrm{~m}$. To transfer the hydrophones on well M345-109 to virtual sources, we cross-correlate the direct P wave arrivals received from entire surface source data set $\left(S_{k}, k=1\right.$ to 149) at well M345-109 with all 
receivers in well M345-408. Then, all cross-correlated traces are summed using following formula (i.e. modified from Alonizi, 2010).

$$
V S_{W 109,408}(t)=\sum_{m=1}^{N} S_{k w 109}(t) \otimes S_{k w 408}(t)
$$

These steps were applied for each individual receiver on well M345-109 to result in 24 virtual sources. The transformation to virtual source domain is explained by many numerical and experimental researchers (e.g. Bakulin and Calvert, 2006; Korneev and Bakulin, 2006). Unwanted wave-fields (e.g. tube waves) have been removed by applying a muting gate based on first arrival wavelets (i.e. the down going P-wave). The deepest virtual sources (i.e. from $340 \mathrm{~m}$ to $400 \mathrm{~m}$ ) were suppressed because its radiation pattern created an ambiguous up-going ray path for VSM with incorrect velocities. Some 82 down-going rays have been used to create a velocity tomogram (see Figure 2).

The near well velocity distribution obtained by the virtual source and ZVSP methods are highly reasonable when compared with lithology obtained from gamma wire-line logs in M345-109 (see figure 3).

\section{Conclusions:}

Simultaneous VSP experiments were completed in two ground water monitoring wells at the Mirrabooka Trial Aquifer Storage and Recovery site; Perth Western Australia. The data from this experiment was used to compare the virtual source and zero offset vertical seismic profiling methods for recovering velocity distribution in the near well setting. Both methods provided high resolution images expressing the heterogeneous nature of acoustic velocity for aquifer zones in the Leederville formation of the Perth Basin. The virtual source method applied for dual vertical wells proved to be highly promising and provides good motivation to complete new experiments with much higher virtual source coverage and consequently ray density for tomographic imaging between wells.

\section{Acknowledgements}

We thank Adrew Greenwood, Roman Pevzner and Elmar Strobach from the Department of Exploration Geophysics- Curtin University for their suggestions and discussions. 


\section{Vienna |'11}

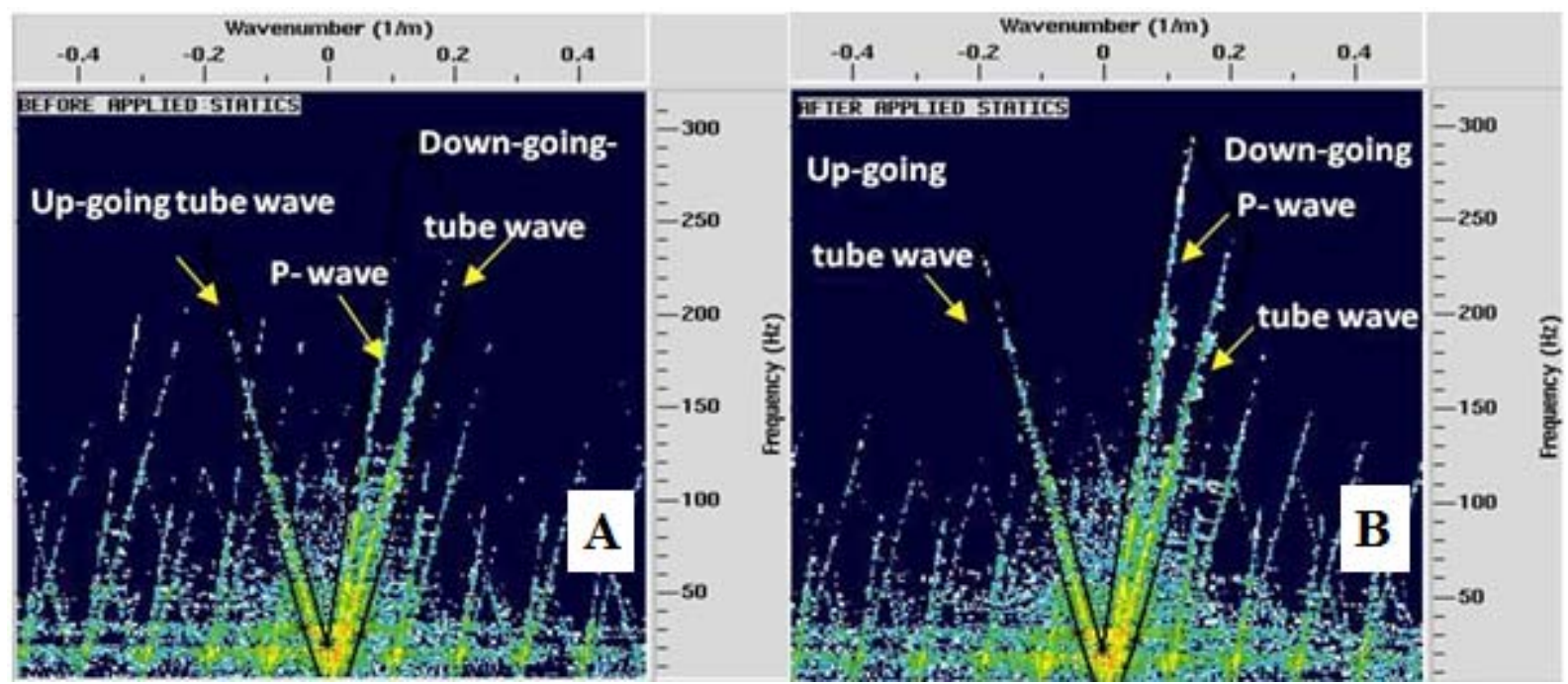

Figure 1A and B F-K domain images for raw (A) and static corrected (B) receiver gathers of ZVSP data at Monitoring well M345-109. Our static corrections significantly improved trends for both tube waves and P-waves. Applying static correction facilitates improved wave-field separation (e.g. tube wave removal). Also notice the considerably improved frequency content after static correction.
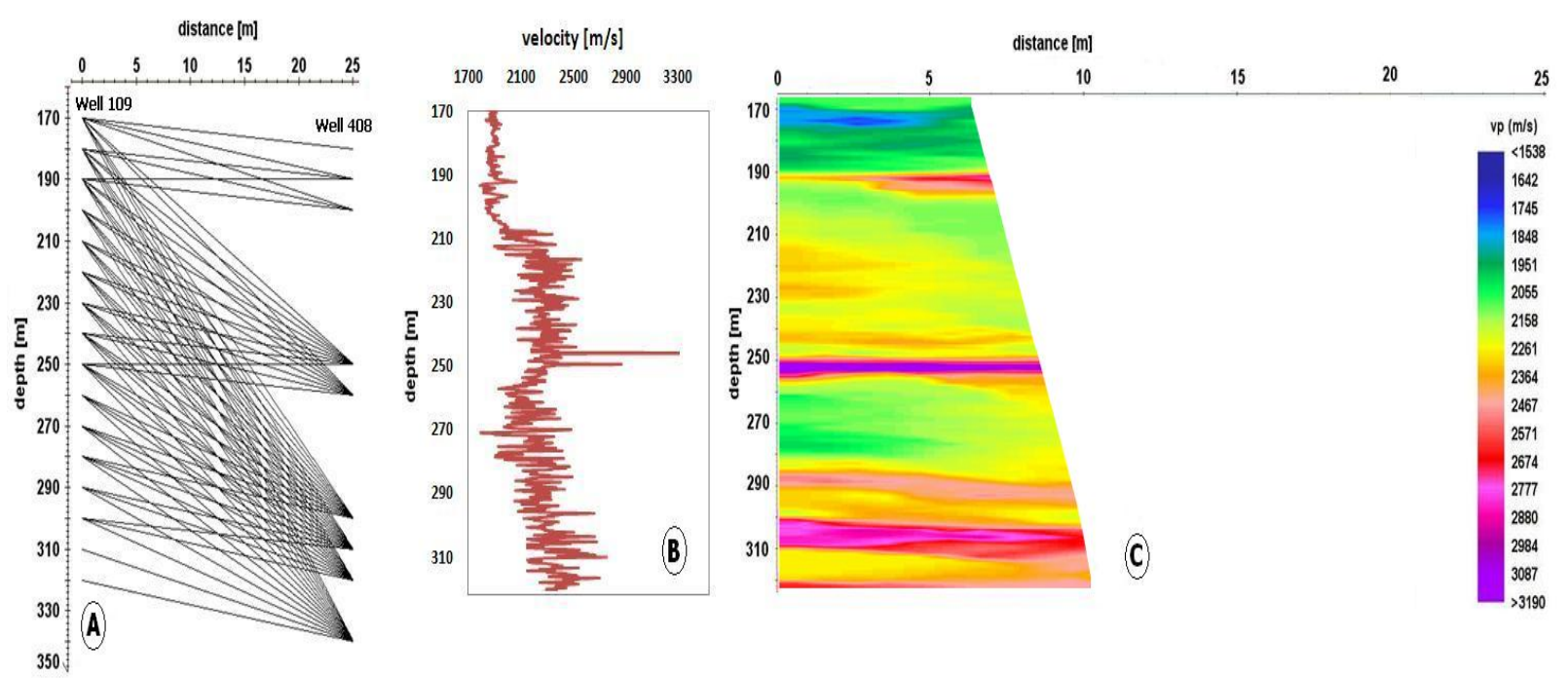

Figure 2A, B and C Images illustrating comparing virtual zone positions, $P$ wave velocities from wire-line logging at $15 \mathrm{KHz}$, and a topographic velocity image obtained from the Virtual Source Method VSM. Figure 2A shows approximate ray coverage using 16 selected virtual sources at well 109. Figure $2 \mathrm{~B}$ shows $\mathrm{P}$-wave velocity acquired at $15 \mathrm{KHz}$ transmitter centre frequency. Figure $2 \mathrm{C}$ shows a tomogram image for the velocity obtained by VSM (only areas with relatively high ray converge are displayed). 


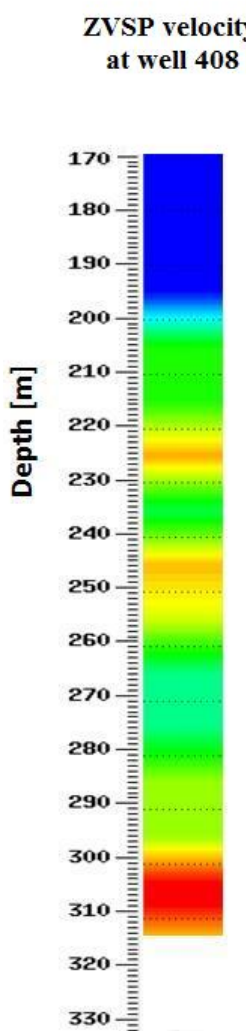

(A)

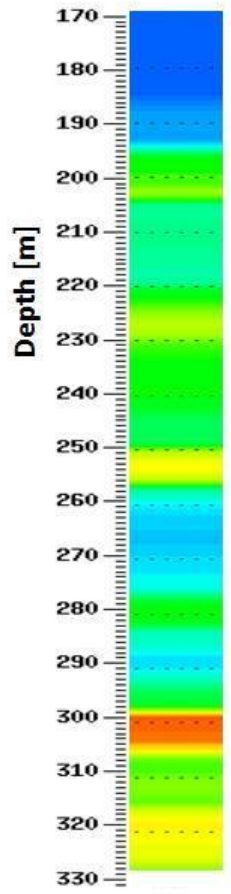

(B)

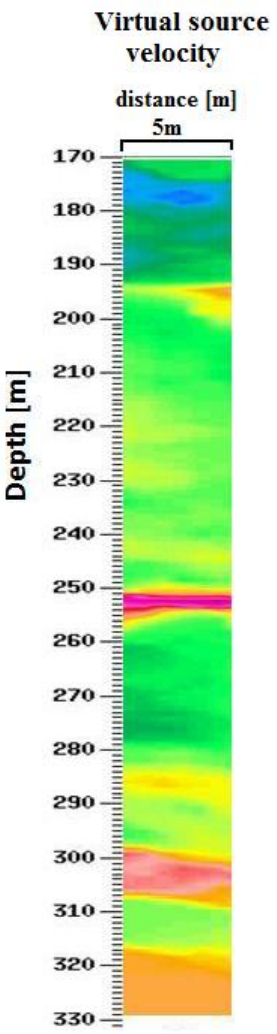

(C)

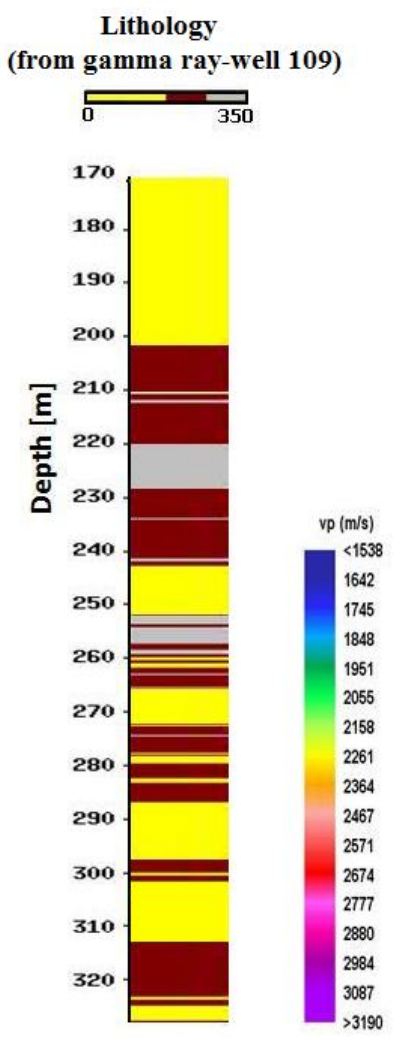

(D)

Figure 3A,B,C and D Comparison of near well velocities obtains from VSM and ZVSP methods. (A) Velocity model obtained at well M345-408 using ZVSP data. (B) Velocity model obtained at well M345-109 using ZVSP data. (C) Near well velocity tomogram obtained by virtual source method. In this tomogram, only areas with high rays coverage is selected for the comparison. (D)Lithology classification using gamma ray wire line logging at borehole M345-109.

\section{References}

Aloniazi, F., Hartley, B. and Pevzner, R. [ 2010]. Numerical Testing of Virtual Source Method. $72^{\text {nd }}$ Annual European Association of Geoscientists and Engineers Conference, Barcelona Spain, 14-18 June 2010

Bakulin, A. and Calvert, R. [2006] The virtual source method: Theory and case study. Geophysics 71(4), SI139-SI150.

Korneev, V. and Bakulin, A. [ 2006] On the fundamentals of the Virtual Source method. Geophysics, 71, A13-A17. 